\title{
Automatic Assignment of the Intrinsically Disordered Protein Tau with 441-Residues
}

\author{
Rhagavendran L. Narayanan, ${ }^{\dagger}$ Ulrich H. N. Dürr, ${ }^{\dagger}$ Stefan Bibow, ${ }^{\dagger}$ Jacek Biernat, ${ }^{\S}$ \\ Eckhard Mandelkow, ${ }^{\S}$ and Markus Zweckstetter, ${ }^{*,+\neq}$ \\ Department of NMR-based Structural Biology, Max Planck Institute for Biophysical Chemistry, Am Fassberg 11, \\ 37077 Goettingen, Germany, DFG Center for the Molecular Physiology of the Brain, 37073 Goettingen, Germany, \\ and Max Planck Unit for Structural Molecular Biology, cloDESY, Notkestrasse 85, 22607 Hamburg, Germany
}

Received June 28, 2010; E-mail: mzwecks@gwdg.de

\begin{abstract}
Intrinsically disordered proteins carry out many important functions in the cell. However, the lack of an ordered structure causes dramatic signal overlap and complicates the NMR-based characterization of their structure and dynamics. Here we demonstrate that the resonance assignment of 441-residue Tau and its smaller isoforms, htau24 (383 residues) and htau23 (352 residues), three prototypes of intrinsically disordered proteins, which bind to microtubules and play a key role in Alzheimer disease, can be obtained within 5 days by a combination of sevendimensional NMR spectra with optimized methods for automatic assignment. Chemical shift differences between the three isoforms provide evidence for the global folding of Tau in solution.
\end{abstract}

In the past decade intrinsically disordered proteins (IDPs) obtained significant attention as more than $30 \%$ of eukaryotic proteins comprise unstructured regions larger than 50 consecutive residues. ${ }^{1}$ A hallmark of IDPs is the lack of a well-structured threedimensional fold. ${ }^{2}$ Despite the absence of a rigid secondary or tertiary structure, IDPs carry out many important functions in the cell, for example the regulation of transcription and translation, the storage of small molecules, and the regulation of the self-assembly of large multiprotein complexes. ${ }^{3}$ Tau, a prototype of IDPs, binds to microtubules and plays a key role in Alzheimer disease. ${ }^{4}$

Since disordered proteins tend to be highly flexible and have variable conformations, they have mostly not been amenable for structure analysis by crystallography. Thus NMR spectroscopy is the only method that allows a description of their conformations and dynamics with high resolution. ${ }^{5}$ The lack of an ordered structure, however, causes dramatic signal overlap limiting previous NMR investigations of IDPs to around 200 residues (e.g., refs 6, 7 ). For the largest Tau isoform with 441 residues, htau40 (Figure 1 ), the number of overlapping signals is 3.5 -fold higher than that in the largest currently assigned globular protein. ${ }^{8}$ In addition, the large number of proline residues, the strongly repetitive primary sequence in the repeat domains, and the complexity of the sequence further complicate the resonance assignment. ${ }^{8,9}$ Only as part of an ongoing project taking several months to years and building on the assignment of smaller fragments of Tau in a "divide-andconquer" strategy, we were recently able to assign the backbone resonances of the full-length protein. ${ }^{8}$

Here we demonstrate that the resonance assignment of 441residue Tau and its smaller isoforms, htau24 (383 residues) and htau23 (352 residues) (Figure 1), can be obtained within 5 days by a combination of seven-dimensional NMR spectra with optimized

\footnotetext{
Max Planck Institute for Biophysical Chemistry.

Max Planck Unit for Structural Molecular Biology.

DFG Center for the Molecular Physiology of the Brain.
}

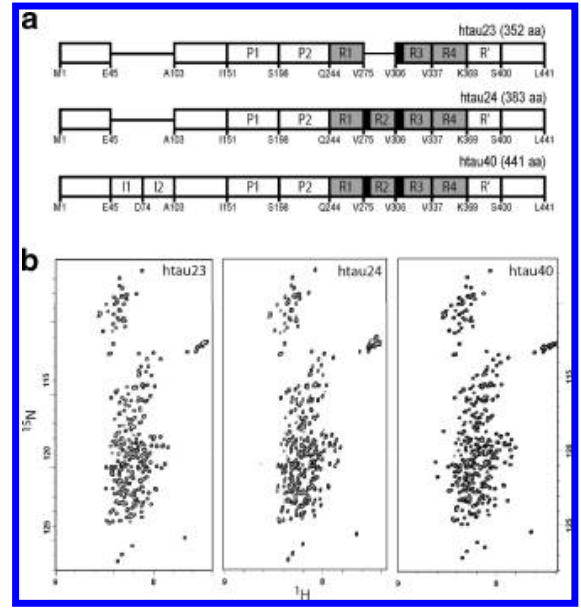

Figure 1. Domain organization (a) and ${ }^{1} \mathrm{H},{ }^{15} \mathrm{~N}$ HSQC spectra (b) of the three Tau isoforms htau23 (no inserts and 3 repeats R1, R3, R4), htau24 (no inserts I1, I2 and 4 repeats) and htau40 (441 residues).

methods for automatic assignment. Chemical shift differences between the three isoforms provide evidence for the global folding of Tau in solution.

Due to the lack of stable hydrogen bonds, NMR spectra of IDPs are sensitive to solvent exchange. At pH 6.8 an increase in temperature from 5 to $35{ }^{\circ} \mathrm{C}$ results in the broadening of about $20 \%$ of backbone resonances of Tau beyond detection in $2 \mathrm{D}{ }^{1} \mathrm{H},{ }^{15} \mathrm{~N}$ HSQC spectra (Supporting Information, Supporting Figure 1). This motivated the use of $\mathrm{H} \alpha$-detection and direct carbon detection for the assignment of IDPs. ${ }^{7,10,11}$ However, when we reduced the $\mathrm{pH}$ to 6.0 the ${ }^{1} \mathrm{H} /{ }^{15} \mathrm{~N}$ signals of Tau were not attenuated at higher temperatures but rather increased by about $40 \%$ due to reduced amide proton exchange, more rapid tumbling, and improved relaxation properties. We thus asked if the favorable relaxation properties help to acquire very highdimensional NMR spectra.

Previously, we obtained the backbone resonance assignment of Tau using 3D HACANNH and 3D HNN experiments at $5{ }^{\circ} \mathrm{C}, \mathrm{pH}$ 6.8. ${ }^{8,12,13}$ Here we recorded 5D HACACONH and 7D HNCO(CA)CBCANH experiments at $25{ }^{\circ} \mathrm{C}, \mathrm{pH}$ 6.0. To avoid prohibitively long measurement times, we employed simultaneous evolution of multiple indirect dimensions ${ }^{14}$ as proposed in automatic projection reconstruction spectroscopy (APSY). ${ }^{15}$ The 7D APSY $\mathrm{HNCO}(\mathrm{CA}) \mathrm{CBCANH}$ correlates in a single cross peak seven different chemical shifts $\left(\mathrm{H}^{\mathrm{N}}(\mathrm{i}), \mathrm{N}(\mathrm{i}), \mathrm{CO}(\mathrm{i}-1), \mathrm{C}^{\beta}(\mathrm{i}-1), \mathrm{C}^{\alpha}(\mathrm{i}-1)\right.$, $\left.\mathrm{N}(\mathrm{i}-1), \mathrm{H}^{\mathrm{N}}(\mathrm{i}-1)\right)$ and sequentially connects residues through $\mathrm{N}$ and $\mathrm{H}^{\mathrm{N}}$ frequencies (Supporting Figure 2). ${ }^{16}$ Due to the high dimensionality of the experiment, signal overlap was reliably resolved and 325 cross peaks were automatically picked by the GAPRO algorithm. ${ }^{15}$ 


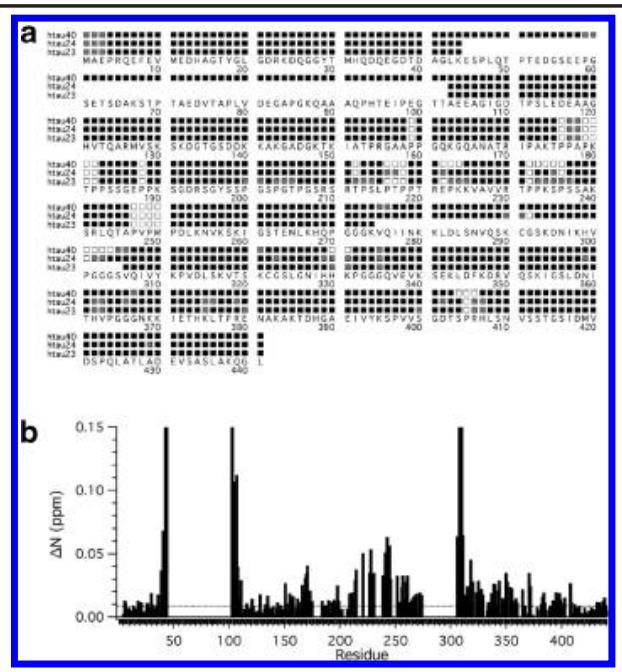

Figure 2. (a) Automatically obtained assignment of Tau isoforms. Black, gray, and white boxes mark assigned, tentatively assigned, and unassigned residues, respectively. Proline chemical shifts were obtained from the 5D APSY from succeeding residues. (b) ${ }^{15} \mathrm{~N}$ chemical shift differences between htau40 and htau23. (--) root-mean-square deviation in ${ }^{15} \mathrm{~N}$ chemical shifts between 7D and 5D APSY.

The 7D HNCO(CA)CBCANH relies on the observation of two sequential $\mathrm{H}^{\mathrm{N}}$ chemical shifts. Proline and residues following proline cannot be observed. This is especially problematic in the prolinerich regions $\mathrm{P} 1$ and $\mathrm{P} 2$ of Tau (22 prolines out of 93 residues). In contrast, proline succeeding residues are observed in the 5D APSY HACACONH ${ }^{15}$ resulting in 391 automatically picked cross peaks with $\mathrm{H}^{\alpha}(\mathrm{i}-1), \mathrm{C}^{\alpha}(\mathrm{i}-1), \mathrm{CO}(\mathrm{i}-1), \mathrm{N}(\mathrm{i}), \mathrm{H}^{\mathrm{N}}(\mathrm{i})$ chemical shift information (Supporting Information, Table $\mathrm{S} 1$ ).

To merge the chemical shift information from the 5D and 7D experiments, deviations between the average values of the chemical shifts in the peak lists of the two spectra are removed. Next identical spin systems from the two experiments are identified. Two spin systems are deemed identical if the deviations between the common chemical shifts $\mathrm{H}(\mathrm{i}) / \mathrm{N}(\mathrm{i}) / \mathrm{CO}(\mathrm{i}-1) / \mathrm{Ca}(\mathrm{i}-1)$ do not exceed defined thresholds. The threshold values were obtained by a fourdimensional grid search maximizing the number of unique matches between the 7D and 5D experiment. With threshold values of 0.033 , 0.043, 0.030, and $0.067 \mathrm{ppm}$ for $\mathrm{H}(\mathrm{i}), \mathrm{N}(\mathrm{i}), \mathrm{CO}(\mathrm{i}-1)$, and $\mathrm{Ca}(\mathrm{i}-1)$, respectively, 294 single matches were obtained. Together with unmatched spin systems from both spectra the merged 7D/5D peak list contained 416 spin systems.

The key step for the automated analysis of the Tau protein is the sequence-specific assignment of the 416 merged spin systems to the primary sequence. Because of the high dimensionality and the large number of spin systems manual assignment is very difficult. In contrast, the high quality of the peak lists makes them suitable for analysis by automatic assignment methods. Here we extended and improved the algorithm of the automatic assignment software MARS: ${ }^{17}$ (i) $\mathrm{H}^{\mathrm{N}}$ and $\mathrm{N}$ connectivity information was implemented and optimized, (ii) random coil chemical shifts were adjusted, and (iii) automatic chemical shift referencing was introduced. ${ }^{18,19}$

Subjecting the merged 7D/5D peak list of 441-residue htau40 to the new MARS algorithm resulted in the automatic assignment of $92 \%$ of assignable residues in less than $3 \mathrm{~h}$ (Figure 2a). For htau24 (383 residues) and htau23 (352 residues) we also recorded 5D HACACONH and 7D HNCO(CA)CBCANH experiments and subjected them to automatic peak picking, merging of spin systems, and assignment: $95 \%$ and $97 \%$ of assignable residues were assigned, respectively (Supporting Information, Table S1).
In addition, we tested if we can extend the "divide-and-conquer" strategy, which was previously used successfully for manual assignment, ${ }^{8}$ to automatic assignment. To this end, we automatically merged the assigned 7D/5D peak list of htau23 with the unassigned 7D/5D peak list of htau40, fixed for single matches (see above) the assignment of peaks of htau40 to those obtained for htau23, and asked MARS to complete the assignment of the remaining peaks of htau40. This increased the assignment of htau40 to $93 \%$. Comparison with the previously established manual assignment of htau 40 at $5{ }^{\circ} \mathrm{C}, \mathrm{pH} 6.8$, shows a nearly perfect match (Supporting Figure 3). The few missing assignments are primarily located in the proline-rich region P1 (Figure $2 a)$, especially for residues in between two prolines. If required, these can be assigned using a 3D HACANNH or a 3D HACAN experiment, which does not rely on detection of amide protons (Supporting Figure 4). ${ }^{20}$

In summary, we demonstrated that the backbone resonance assignment of very large disordered proteins can be determined by automatic assignment within 5 days of measurement time and a few hours of calculation reducing the overall analysis time by more than an order of magnitude. In addition, $\mathrm{CO}$ and $\mathrm{C}^{\beta}$ chemical shifts are obtained, which are difficult to obtain from a manual analysis due to signal overlap, but which are important for analysis of structural propensities. The assignment of the three different isoforms of Tau established in this study at $25{ }^{\circ} \mathrm{C}$ allows investigation of isoform specific differences in the structure, microtubule binding, and aggregation of Tau. ${ }^{15} \mathrm{~N}$ chemical shift differences between Tau isoforms are not restricted to the segment borders, providing evidence for the global folding of Tau in solution (Figure 2b and Supporting Figure 5).

Acknowledgment. We thank S. Hiller, C. Griesinger, R. Bajaj, the VW foundation, the Max Planck Society, and the DFG (Heisenberg scholarship ZW 71/2-2 and 3-2 to M.Z.) for support.

Supporting Information Available: Assignment statistics and chemical shift differences for Tau isoforms. This material is available free of charge via the Internet at http://pubs.acs.org.

\section{References}

(1) Dunker, A. K.; Silman, I.; Uversky, V. N.; Sussman, J. L. Curr. Opin. Struct. Biol. 2008, 18, 756-764.

(2) Fink, A. L. Curr. Opin. Struct. Biol. 2005, 15, 35-41.

(3) Dyson, H. J.; Wright, P. E. Nat. Rev. Mol. Cell Biol. 2005, 6, 197-208.

(4) Braak, H.; Braak, E. Acta Neuropathol. (Berlin) 1991, 82, 239-259.

(5) Dyson, H. J.; Wright, P. E. Chem. Rev. 2004, 104, 3607-3622.

(6) Perez, Y.; Gairi, M.; Pons, M.; Bernado, P. J. Mol. Biol. 2009, 391, 136.

(7) Csizmok, V.; Felli, I. C.; Tompa, P.; Banci, L.; Bertini, I. J. Am. Chem. Soc. 2008, 130, 16873-16879.

(8) Mukrasch, M. D; Bibow, S.; Korukottu, J ; Jeganathan, S.; Biernat, J.; Griesinger, C.; Mandelkow, E.; Zweckstetter, M. PLoS Biol. 2009, 7, e34.

(9) Smet, C.; Leroy, A.; Sillen, A.; Wieruszeski, J. M.; Landrieu, I.; Lippens, G. ChemBioChem 2004, 5, 1639-1646.

(10) Bermel, W.; Bertini, I.; Felli, I. C.; Lee, Y. M.; Luchinat, C.; Pierattelli, R. J. Am. Chem. Soc. 2006, 128, 3918-3919.

(11) Mantylahti, S.; Aitio, O.; Hellman, M.; Permi, P. J. Biomol. NMR 2010, 47, 171-181.

(12) Panchal, S. C.; Bhavesh, N. S.; Hosur, R. V. J. Biomol. NMR 2001, 20, $135-147$.

(13) Zweckstetter, M.; Bax, A. J. Am. Chem. Soc. 2001, 123, 9490-9491.

(14) Atreya, H. S.; Eletsky, A.; Szyperski, T. J. Am. Chem. Soc. 2005, 127, 4554-4555.

(15) Hiller, S.; Fiorito, F.; Wuthrich, K.; Wider, G. Proc. Natl. Acad. Sci. U.S.A. 2005, 102, 10876-10881.

(16) Hiller, S.; Wasmer, C.; Wider, G.; Wuthrich, K. J. Am. Chem. Soc. 2007, 129, 10823-10828.

(17) Jung, Y. S.; Zweckstetter, M. J. Biomol. NMR 2004, 30, 11-23.

(18) Schwarzinger, S.; Kroon, G. J.; Foss, T. R.; Chung, J.; Wright, P. E.; Dyson, H. J. J. Am. Chem. Soc. 2001, 123, 2970-2978.

(19) Wang, L.; Eghbalnia, H. R.; Bahrami, A.; Markley, J. L. J. Biomol. NMR 2005, 32, 13-22.

(20) Kanelis, V.; Donaldson, L.; Muhandiram, D. R.; Rotin, D.; Forman-Kay, J. D.; Kay, L. E. J. Biomol. NMR 2000, 16, 253-259.

JA105657F 